\title{
GUT MICROBIOME PROFILE IN ZINC DEFICIENT INFANTS USING NEXT GENERATION SEQUENCING
}

\section{Mehwish Durrani $^{1 凶}$, Rubina Nazli', Nabila Sher', Muhammad Abubakr², Johar Ali}

\begin{abstract}
OBJECTIVE: To find out the self-motivated cadence of intestinal microbiota by zinc obtainability with help of next generation sequencing.

METHODS: This cross-sectional study was conducted on infants aging I-4 months from Peshawar, Pakistan. Out of 50 infants enrolled in study, data of 46 healthy infants [23 breast-fed (BF) and 23 formula-fed (FF)] were available for final analysis. Their blood and stool samples were collected and analyzed for zinc and fecal DNA extraction, for metagenomic shot gun sequencing using next generation.
\end{abstract}

RESULTS: Mean age of infants was $78.40 \pm 35.88$ days. Actinobacteria, Bacteroidetes, Firmicutes and Proteobacteria are the four dominating major phyla found in the gut microbiota of the sample population. In breast-fed infants, mean plasma zinc was $0.138 \pm 0.118 \mathrm{mg} / \mathrm{L}$ whereas, in formula-fed infants it was $0.120 \pm 0.026 \mathrm{mg} / \mathrm{L}$. Correlation of zinc levels with gut microbes revealed that Phylum Bacteroidetes was very low in breast-fed while in formula-fed infants it was negligible to an extent that cannot be correlated with zinc. It was also observed that there is abundance of Proteobacteria ( $B F: r=-0.276, p=0.264$ ) and (FF: $r=-0.043, p=0.873$ ) in both the zinc deficient groups whereas, Firmicutes content was (BF: $r=0.392, p=0.207)$ and ( $F F: r=-0.030, p=0.905)$.

CONCLUSION: Both breast-fed and formula-fed infants had low gut microbial diversity due to low zinc levels emphasizing the need to address and rectify this problem. The improved zinc status of the infants will enable the researchers to establish and ascertain the association of zinc status with the diversity of microbiome.

KEY WORDS: Plasma zinc (Non-MeSH); Gut Microbiota (MeSH); Breast feeding (Non-MeSH), Formula-fed infants (Non-MeSH).

THIS ARTICLE MAY BE CITED AS: Durrani M, Nazli R, Sher N, Abubakr M, Ali J. Gut microbiome profile in zinc deficient infants using next generation sequencing. Khyber Med Univ J 202I;I3(I):4-9. DOI: https://doi.org/ 10.35845/kmuj.2021.20739.

\section{INTRODUCTION}

nfant gut at the time of birth immediately get colonized by the microbes during the process of birth, although it is now known that in utero, the environment is not completely sterile. Infants' microbial colonization is a highly dynamic process as it is affected from the environment to which they are exposed. Among the colonizers of infants' gut, mother-microbes are the first one that shape and seeds infants' intestine.' Infants' intestinal microbiome are contributed from the mother's vagina, skin, stool and then mother milk. Mother milk, human milk oligosaccharides, and infant's microbiome are linked in a triad with one another. In breast-fed infants, mother milk microbes have an important role in the formation of intestinal microflora. On the other hand formula milk is deprived of maternal antibodies, complex oligosaccharides and skin microbes. ${ }^{3}$ Studies have reported that skin related taxa like Staphylococcus, Corynobacteria, Gemella and. Propionibacterium were the most predominant and consistent candidates of breast-fed infants which signifies the more intense and frequent contact of mothers skin with the breast-fed infants. ${ }^{4}$ The gut microbiome of breast-fed and formula-fed infants differs greatly. During the first six months of life, the relative abundances of Firmicutes and Bacteroidetes, carbohydrates metabolic pathways facilitated by microbes and overall
I: Institute of Basic Medical Sciences, Khyber Medical University, Peshawar, Pakistan.

2: Pak International Medical College, Peshawar, Pakistan

3: Center of Genomics, Rehman Medical Institute, Peshawar, Pakistan.

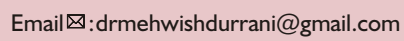

Contact \#: +92-333-9313716

Date Submitted: August 25, 2020

Date Revised: March 03, 2021

Date Accepted: March 23, 202I

bacterial diversity continuously increases in breast-fed as compared to non-breastfed infants. ${ }^{2}$ Literature supports the fact that bacterial species richness is more pronounce in non breast-fed infants than in breast-fed infants.

Large gut is the main reservoir that inculcate largest count of microbes in humans as compared to other bacterial recess like mouth, skin, ear and vagina. ${ }^{6}$ Following birth, the human milk is the main modulator of the gut microbial colonization. ${ }^{7}$ Alpha diversity is lower in breast-fed than in formula-fed infants, and at the age of 3-4 months on formula the infants had high microbial diversity. ${ }^{8}$ The gut microbiome of an infant start maturing with the initiation of breast feeding as mother milk inoculate new microbial communities within the gut of neonates. ${ }^{9}$ The formula milk is concerned to impair the immune system of an infant and develops metabolic syndromes in adult life. ${ }^{10}$ It is evident from literature that the breast milk microbiome varies from colostrum to late lactational phases, maternal health status and gestational age." Few dominating genera in breast milk are Streptococcus, Staphylococcus, Pseudomonas, Serratia, Ralstonia, Corynebacterium, Propionibacterium, Bradyrhizobiaceae, and Sphingomonas. ${ }^{12}$ It has been observed that human milk oligosaccharides act as prebiotics and nourish the specific microbial colonies of Bifidobacterium in infant gut, they inhibit the growth of pathogenic organisms enhancing inflammatory and immunological responses. ${ }^{13}$ Formula feeding is associated with high bacterial diversity, increased count of Provetella, C.defficil, E.coli and Bacteroides fragilis and 
decrease count of Bifidobacterium. ${ }^{14}$

Zinc is an essential mineral for cellular functions. Over 300 known enzymes and approx. 1000 transcription factors are dependent on zinc for their proper functioning. ${ }^{15}$ It has got role in maturation of immune system, part of genetic enzymes, role in keratinization and also serves as a cofactor in many enzymatic processes. $^{16}$ Zinc also plays important role in various biochemical pathways, affecting majority of the organ systems like gastrointestinal tract, central nervous system, skin, reproductive system and skeletal system requiring an optimum content of zinc in the body. The causes of zinc deficiency can be dietary, increased losses and inadequate absorption. In infants, zinc deficiency is manifested by increase episodes of pneumonia, diarrhea and malaria. ${ }^{17}$ For 0-6 months infants the daily dietary requirement for zinc is $2 \mathrm{mg} / \mathrm{d}$ at the age of 7-12 months it is increased two folds. ${ }^{18}$ For newborns and infants up to six months of life breast milk is considered to be a complete nutrition as it provides essential nutrients for infants growth and development. ${ }^{19}$ During early phases of lactation, the zinc concentration is highest in the breast milk and starts declining until the $3^{\text {rd }}$ month of infancy. Due to this limiting level during first 3 months, it has been observed that zinc is the first limiting nutrient in human breast milk. ${ }^{20}$ There is scarce information available in the literature as to whether these limited levels of zinc have any impact on the gut microbiome. Therefore, this study was undertaken to determine the relationship, if any, between the zinc status and diversity of gut microbiome in infants from Peshawar, Khyber Pakhtunkhwa Pakistan and to compare the gut microbial metabolism with European cohort.

\section{METHODS}

This was a cross-sectional study, carried out in Peshawar, Khyber Pakhtunkhwa Pakistan. The study was approved by the Khyber Medical University Ethics board (KMU-Ethics Board) on 27th of October 2016 for a period of 4 years under the study reference number DIR/KMU$\mathrm{EB} / \mathrm{PZ} / 000316$. This study was started in October 2017 and ended in March 2018.

Based on median difference of 14.3 $\mathrm{mmol} / \mathrm{kg}$ total short chain fatty acids between breast-fed and formula-fed infants at 2 months of age and considering margin of error of 5\%, total of 25 infants are required in each group (total $n=50$ ). The study was explained to the mothers in local language. The mothers of all the participants gave written informed consent.

Healthy infants, aging I-4 months were enrolled in this study having no clinical sign of any illness (Figure I). They were predominantly either on breast feeding or formula feeding. Each patient was screened using a health check questionnaire to rule out conditions related to changes in physiology of the gut which can alter gut microbiota composition and metabolic activity such as medications, gut surgeries, systemic use of prebiotics and probiotics, specifically exclusion criteria were: use of antibiotics in the past 4 weeks (infants as well a feeding mothers), infants having persistent diarrhea , systemic use of pre- or probiotics (defined as a daily use of prebiotics or probiotics). The use of certain complex polysaccharides (prebiotics) or live cultures of bacteria (probiotics) have also been shown to alter the metabolic activity and gut microbial diversity. Therefore, infants who were prescribed with or using probiotic were excluded from the study.

\section{Anthropometric assessment of} enrolled infants and mothers

Weight and length measurement of an infant was performed once. Infant crown-heel length was measured once by using headpiece and foot piece both applied perpendicular to the hard surface and non-stretch tape was used to measure the length in centimeters. Head circumference was measured at a level passing from supraorbital protuberance anteriorly and occipital protuberance posteriorly using non-stretch tape to the nearest $0.1 \mathrm{~cm}$. Infant's weight was determined by using Beurer Digital Baby Scale (BY-80) in kilograms. Height of the mothers was measured with a portable stadiometer with the head in horizontal Frankfort plane. Mid upper arm circumference was measured to the nearest $0.1 \mathrm{~cm}$ using a plastic measuring tape. Weight of the mother was measured by Beurer digital scale "GS 200 Allium" to the nearest $100 \mathrm{gm}$.

\section{Mothers of the infants}

Only mothers of the recruited participants were included in this study. The aim of their optional recruitment was to analyze their plasma zinc levels and then look for effect of zinc deficient and zinc sufficient state on gut microbiota and differences of bacterial community structure and metabolic activity with their infants (breast-fed infants). Dietary information was also collected from the mothers through 24-hour dietary recall (food intake during $24 \mathrm{hrs}$ ).

\section{Blood sampling and stool sampling}

The blood samples and the fecal sample of the infants were taken and subjected to plasma zinc assay, Perklin-Elmer double beam atomic absorption spectrophotometer, model AAS-700, with a deuterium lam for background correction was used to measure concentration of Zinc. Fecal DNA extraction was done by following the standard protocols using Qigen ministool kit [not correct], the next generation sequencing was carried out by Illumina MiSeq sequencer ${ }^{\circledR}$. Shot gun metagenomic sequencing was performed to analyze the bacterial population. All the metagenomic work and analyses were carried out at Rehman Medical Institute (RMI) genomic center, Peshawar, Pakistan.

\section{Next generation sequencing}

Microbiome Helper Standard Operating procedures were followed to process the shot gun metagenomic data. The raw metagenomic reads were examined by using Fast QC tool. To ensure the quality control on metagenomic sequencing data, knead data tool was applied to trim the content of host genome to exclude the high ratio of host to bacterial reads. This tool was applied to perform in silico separation of bacterial reads from contaminated host reads. Read trimming and filtering of low-quality reads was done through Trimmomatic tool. As it is an efficient preprocessing, which could correctly handle paired-end data. ${ }^{20}$ Bowtie2 is an ultrafast and memory efficient tool for aligning sequencing reads to long reference reads. It supports gapped, local, and paired-end alignment modes. ${ }^{21}$ MetaPhalan2, a computational tool for profiling the composition of microbial communities from metagenomic shot gun sequencing data. For taxonomic profiles, the processed reads 
TABLE I: CORRELATION OF PLASMA ZINC WITH DIFFERENT TAXONOMIC RANKS OF GUT MICROBIOTA

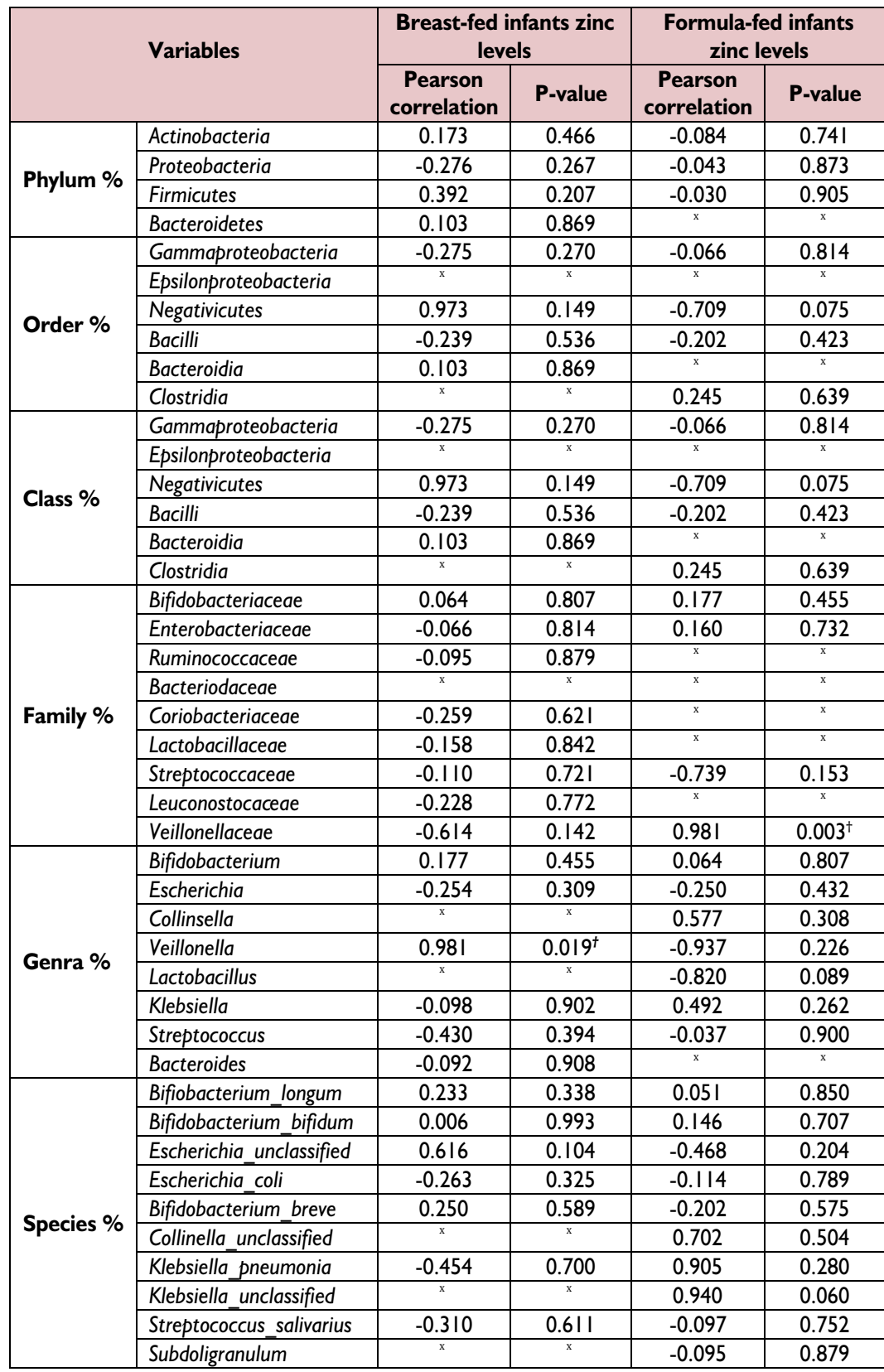

${ }^{x}$ the microbes were not present in the detectable amounts and hence could not be correlated with zinc level

were further subjected to MetaPhalan2. A unique clade-specific marker is used to detect the taxonomic clades present in samples of microbiome and estimating the relative abundance of microbiome. ${ }^{22}$

\section{Statistical analysis}

All the results of microbes from NGS analysis were arranged and organized on Excel sheet along with plasma zinc of infants from both the groups. Concentra-tion of gut microbiota was expressed in percentage of microbes. Zinc levels in BFI were found to be $0.138 \pm 0.118 \mathrm{mg} / \mathrm{L}$ whereas in FFI it was observed to be $0.1200 \pm$ $0.026 \mathrm{mg} / \mathrm{L}$. Descriptive statistics was applied for comparison between the groups and to find out correlation and association of plasma zinc of infants with got microbes. All the data were correlation was applied to explore any association and correlation of plasma expressed as mean \pm SD. Pearson zinc levels of infants with gut microbial diversity in both the groups at all levels. $\mathrm{P}$-values of less than 0.05 were considered significantly different.

\section{RESULTS}

Out of 75 infants identified, fifty infants were enrolled in study. Data of 46 infants were available for final analysis (figure I). Mean age of infants was $78.40 \pm 35.88$ days.

Overall phylogenetic profile of the gut microbiota in Pakistani healthy infants

Overall a total number of 55, 08374 sequencing reads were generated with an average of I, I 4757 reads per sample were analyzed and were classified into Phyla-Species level taxa.

Gut microbiota Profile in breast-fed and formula-fed infants

It was observed that at Phylum levels the two groups in case of Actinobacteria and Proteobacteria were not significantly different. Whereas, Firmicutes in both the groups significantly vary with $p=0.00$ I. Which means that formula-fed infants harbour more Firmicutes than breast-fed infants. On the other hand, Bacteroides were marginally significant with $p=0.098$ in both the groups. When class of the microbes were compared between the groups it was found that Actinobacteria, Gammaproteobacteria, Negativicutes were not significantly different in both the groups. However, Bacilli were significantly higher $(p=0.007)$ in formula-fed infants when compared with 23 breast-fed infants, as shown in our recently published data from the same project. ${ }^{23}$ When order levels microbes were compared between the groups it was observed that Bifidobacteriales were higher insignificantly in formula-fed infants, Enterobacteriales, Coriobacteriales, Lactobacillales, except Coriobacteriales all were higher in formula-fed infants in comparison with breast-fed infants. Nevertheless, Selenomonadales were significantly higher $p=0.037$ in formula-fed infants. While comparing the \% means of Bifidobacteriaceae it was observed that they are insignificantly higher in formula-fed infants. However, Enterobacteriaceae, Coriobacteriaceae and Lactobacillaceae were not significantly different between 


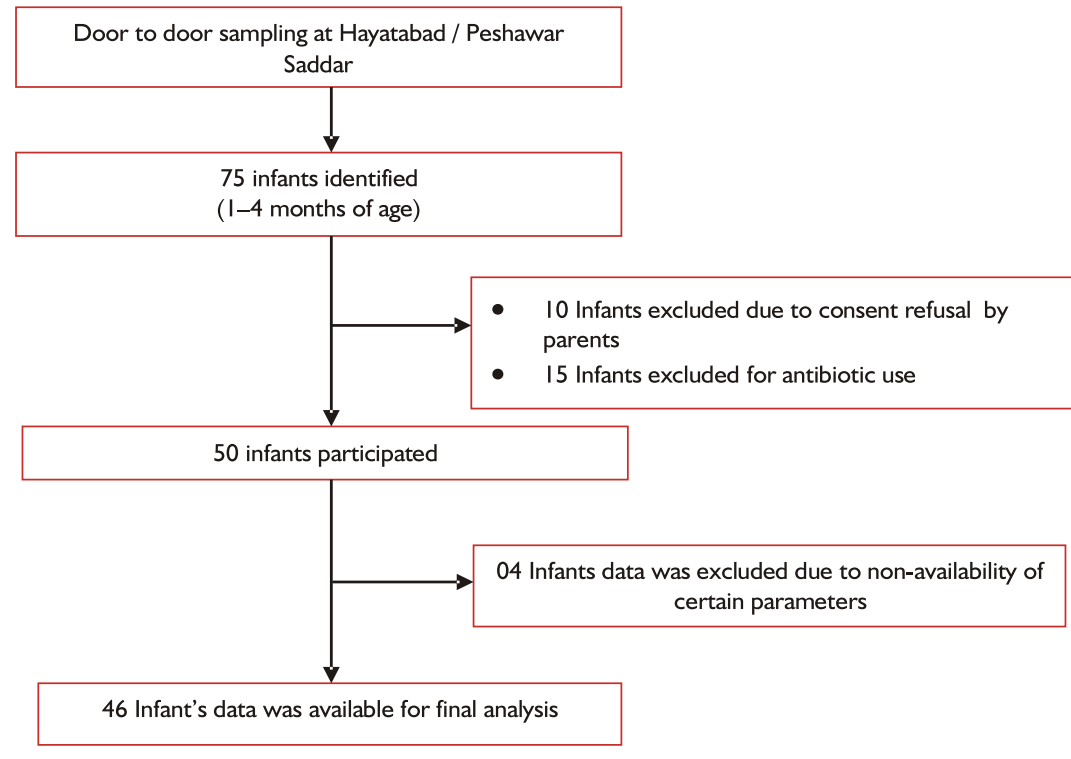

Figure I: Flow chart of participant screening

the two groups. Whereas, Streptococcaceae were significantly higher $p=0.033$ in formula-fed infants. Similarly, Veillonellaceae were also found to be marginally significant $p=0.070$ in formula-fed infants when compared with breast-fed infants. Bifidobacterium and Klebsiella were higher in formula-fed infants but were unable to achieve the level of significance. Escherichia were comparatively higher in breast-fed infants. Wherever, Veillonella and Lactobacillus were almost present equally in both groups. On the other hand, Streptococcus\% were significantly higher $p=0.031$ in formula-fed infants. Bifiobacterium_longum, Bifidobacterium bifidum, Bifidobacterium breve was comparatively similar in both groups. Escherichia_unclassified was insignificantly higher in formula-fed infants. Similarly, Veillonella_unclassified and Klebsiella pneumonia, was also higher in formulafed infants with no significant $p$-value. Presence of Streptococcus salivarius was significantly higher $(p=0.029)$ in formula-fed infants as compared to breast-fed infants (data given in Table II of our recently published article from the same project). ${ }^{23}$

\section{Correlation of Gut microbiota Profile with plasma zinc of infants}

Correlation of plasma zinc of infants were performed at all the levels of gut microbial profile from phyla to species but surprisingly it was observed the correlation at all the levels were insignificant. Correlation and association could not attain the significant level in most of the cases results summarized in table $\mathrm{l}$.

\section{DISCUSSION}

The literature survey reveals that this study is the first attempt to ascertain the relationship between plasma zinc of infants and gut microbiota and to evaluate the effect of plasma zinc levels on gut microbiota. Results showed that all the infants were zinc deficient. The zinc levels in both breast-fed and formula-fed infants were low. The primary risk factors of zinc deficiency are the insufficient dietary zinc intake and poor bioavailability. ${ }^{24}$ The environment of gut microbiome is indispensable for zinc metabolism. Recent literature from animal model revealed that zinc deficiency very badly affect the gut microbial diversity and leads to reduction in overall taxonomic richness. ${ }^{24}$ and Proteobacteria are the four dominating major phyla of gut microbiome. ${ }^{25}$ While analyzing the association of low zinc levels with gut microbes it was observed that phylum Bacteroidetes was found to be very low in both breast-fed and formula-fed infants. Whereas the ratio of Firmicutes to Proteobacteria was also low in both the groups. Similar results were shared by Reed S, et al. in 2015 in a study carried Actinobacteria, Bacteroidetes, Firmicutes out on broiler chick (Gallus gallus). ${ }^{26}$

At family level Bifidobacteriaceae and Enterobacteriaceae were higher in both the groups endorsing the results of Reed S, et al. 2015. ${ }^{26}$ The population of microbial families like Bacteriodaceae, Coriobacteriaceae, Prevotella, Campylobacteriaceae, Streptococcaceae and Veillonellaceae were lesser in breast-fed infants having low levels of plasma zinc. In formula-fed zinc deficient subjects, Streptococcaceae were higher while Ruminococcaceae, Bacteriodaceae, Leuconostocaceae \%, Lactobacillaceae, Lachnospiraceae and Veillonellaceae all were low. Reed et al in $2018^{24}$ reported that zinc sufficient broiler chicks (Gallus gallus) harbor Ruminococcaceae in their gut. Therefore, it can be assumed that zinc insufficiency states lead to Ruminococcaceae deficiency in the gut. At genus level in breast-fed zinc deficient group Bifidobacterium and Escherichia $\%$ were elevated whereas, Collinsella, Veillonella, Enterococcus, Morganella, Akkermansia, Bacteroides and Prevotella all were present in very little amount. The formula-fed zinc deficient group had higher count of Bifidobacterium and lesser number of Escherichia in comparison with Bifidobacterium. However, Veillonella, Streptococcus \%, Bacteroides, Leuconostoc, Subdoligranulum, Megaspheare, Clostridia and Blautia were drastically low in this group. Similar result was reported by Reed S, et al. 2015 regarding Clostridia from their experimental model (Gallus gallus), ${ }^{26}$ other genera not investigated in their study. This can be related to the fact that chick microbiota is not much diverse as compared to humans. At species level Bifiobacterium_longum, Bifidobacterium bifidum were found to be higher in both formula-fed and breast-fed infants, whereas Klebsiella_pneumonia, Klebsiella_unclassified and Streptococcus salivarius were low in comparison with the former. Furthermore, Collinella unclassified, Lactobacillus gasseri, Klebsiella oxytoca, Ruminococcus, Escherichia unclassified, Escherichia coli, Bifidobacterium breve, Veillonella unclassified, Subdoligranulum, Leuconostoc lactis, Megasphaere unclassified, Lactobacilli ruminis were negligibly detected in these zinc deficient infants. Ruminococcus was reported to signify zinc sufficient state by Reed S, et al. in 
$2018{ }^{24}$ This supports our finding that zinc deficiency affects Ruminococcus count.

\section{CONCLUSION}

The findings of this study provide a gateway for direct comparison of gut microbial diversity of infants from Pakistan with European cohort. Our study for the first time disclosed the primary colonizer of healthy infant's gut in Pakistani population by shot gun next generation sequencing. Data of the present study revealed that both breastfed and formula-fed infants had low gut microbial diversity due to low zinc levels emphasizing the need to address and rectify this problem. The improved zinc status of the infants will enable the researchers to establish and ascertain the association of zinc status with the diversity of microbiome. Extensive research is required in humans as they have very diverse microbiome as compared to any other experimental model.

\section{ACKNOWLEDGMENT}

Authors would like to express their appreciation to (late) Dr. Muhammad Jaffar Khan who co-supervised and designed this study. His guidance, suggestion and constant encouragement made it possible to accomplish this research task. We are also very thankful to all the mothers who consented to participate along with their infants in this study.

\section{REFERENCES}

I. Timmerman HM, Rutten NB, Boekhorst J, Saulnier DM, Kortman $\mathrm{GA}$, Contractor N, et al. Intestinal colonisation patterns in breast-fed and formula-fed infants during the first I 2 weeks of life reveal sequential microbiota signatures. Sci Rep 2017 Aug $21 ; 7(\mathrm{I}): 8327$. DOI: $10.1038 / \mathrm{s}$ 41598-017-08268-4.

2. Ho NT, Li F, Lee-Sarwar KA, Tun HM, Brown BP, Pannaraj PS, et al. Metaanalysis of effects of exclusive breastfeeding on infant gut microbiota across populations. Nat Commun $2018 \mathrm{Oct}$ 9;9(I):4I69. DOI: 10.1038/s4I467018-06473-x.

3. Pannaraj PS, Li F, Cerini C, Bender JM, Yang S, Rollie A, et al. Association between breast milk bacterial communities and establishment and development of the infant gut microbiome. JAMA Pediatrics 2017; I7I(7):647-54. DOI: 10.100I/ jamapediatrics.2017.0378.

4. Jiménez E, Delgado S, Maldonado A, Arroyo R, Albújar M, García N, et al. Staphylococcus epidermidis: a differential trait of the fecal microbiota of breast-fed infants. BMC Microbiol 2008;8(I): |43. DOI: I0.II86/I47I2180-8-143.

5. Azad MB, Konya T, Persaud RR, Guttman DS, Chari RS, Field CJ, et al. Impact of maternal intrapartum antibiotics, method of birth and breastfeeding on gut microbiota during the first year of life: a prospective cohort study. $\mathrm{Br} \mathrm{J}$ Obs Gynaecol (BJOG) 2016;123(6):98393. DOI: I0. I I I I/I47I-0528.I360I.

6. Turnbaugh PJ, Ley RE, Hamady M, Fraser-Liggett CM, Knight R, Gordon Jl. The human microbiome project. Nature 2007;449(7I64):804. DOI: 10.1038/nature06244.

7. Civardi E, Garofoli F, Mazzucchelli I, Angelini M, Manzoni P, Stronati M. Enteral nutrition and infections: the role of human milk. Early Hum Dev 2014 Mar;90 Suppl I:S57-9. DOI: 10.10I6/S0378-3782(I4)70019-2.

8. Mueller E, Blaser M. Breast milk, formula, the microbiome and overweight. Nature Rev Endocrinol 2018;14(9):5I0-I. DOI: 10.1038/s 4I574-0।8-0066-5.

9. Palmer C, Bik EM, DiGiulio DB, Relman DA, Brown PO. Development of the human infant intestinal microbiota. PLoS Biol 2007;5(7):e 177. DOI: 10.1371/journal.pbio.00 50177.

10. Gale C, Logan KM, Santhakumaran S, Parkinson JR, Hyde MJ, Modi N. Effect of breastfeeding compared with formula feeding on infant body composition: a systematic review and meta-analysis. Am J Clin Nutr 2012;95(3):656-69. DOI: 10.3945/ ajcn. II I.027284.

II. Khodayar-Pardo P, Mira-Pascual L, Collado MC, Martinez-Costa C. Impact of lactation stage, gestational age and mode of delivery on breast milk microbiota. J Perinatol 2014;
34(8):599-605. DOI: 10.1038/jp. 2014.47.

12. Hunt KM, Foster JA, Forney LJ, Schutte UM, Beck DL, Abdo Z, et al. Characterization of the diversity and temporal stability of bacterial communities in human milk. PLoS One 201I;6(6):e21313. DOI: 10.1371/journal.pone.002/313.

13. Sudo N, Sawamura S, Tanaka K, Aiba Y, Kubo C, Koga Y. The requirement of intestinal bacterial flora for the development of an lgE production system fully susceptible to oral tolerance induction. J Immunol 1997; 159(4): I739-45.

14. Bezirtzoglou E, Tsiotsias A, Welling GW. Microbiota profile in feces of breast- and formula-fed newborns by using fluorescence in situ hybridization (FISH). Anaerobe 20I I;17(6):478-82. DOI: 10.1016/j. anaerobe.201 I.03.009.

15. Watson L, Cartwright D, Jardine LA, Pincus D, Koorts P, Kury S, et al. Transient neonatal zinc deficiency in exclusively breastfed preterm infants. J Paediatr Child Health 20।8;54(3):319-22. DOI: I0.1III/ jpc. 13780 .

16. Aumeistere L, Ciprovica I, Zavadska D, Bavrins K, Borisova A. Zinc Content in Breast Milk and Its Association with Maternal Diet. Nutrients 2018;10(10). DOI: 10.3390/nul0101438.

17. Arýca S, Arýca V, Dag H, Kaya A, Hatipoglu $S$, Fenercioglu $A$, et al. Serum zinc levels in children of 0-24 months diagnosed with pneumonia admitted to our clinic. Int J Clin Exp Med 20I I;4(3):227-33.

18. Ackland ML, MichalczykAA. Zinc and infant nutrition. Arch Biochem Biophys 2016;611:51-7. DOI: 10.1016/j.abb.2016.06.01।.

19. Solomons NW, Vossenaar M. Nutrient density in complementary feeding of infants and toddlers. E J Clin Nutr 2013;67(5):50I-6. DOI: 10.1038/ejcn.2013.46.

20. Bolger AM, Lohse M, Usadel B. Trimmomatic: a flexible trimmer for Illumina sequence data. Bioinfor- 
matics 20 I4;30(I5):2 I I4-20.

21. Langmead B, Salzberg SL. Fast gapped-read alignment with Bowtie 2. Nat Methods 2012;9(4):357-9. DOI: I0.1038/nmeth. 1923.

22. Truong DT, Franzosa EA, Tickle TL, Scholz M, Weingart G, Pasolli E, et al. MetaPhIAn2 for enhanced metagenomic taxonomic profiling. Nat Methods 2015; 12(10):902-3. DOI: 10.1038/nmeth.3589.
23. Durrani M, Nazli R, Fatima S, Abubakr M. Impact of feeding practice on diversity pattern of the gut microbiomein infants. J Ayub Med Coll Abbottabad 2020;32(4):55I 7.

24. Reed S, Knez M, Uzan A, Stangoulis JCR, Glahn RP, Koren O, et al. Alterations in the Gut (Gallus gallus) Microbiota Following the Consumption of Zinc Biofortified Wheat (Triticum aestivum)-Based Diet. J Agric Food Chem 20 I 8;66(25):629।9. DOI: I0.102 I/acs.jafc.8b0 I48I.
25. Del Chierico F, Vernocchi P, Petrucca A, Paci P, Fuentes S, Praticò G, et al. Phylogenetic and metabolic tracking of gut microbiota during perinatal development. PLoS One 2015; I0(9): e0137347. DOI: 10.1371/journal. pone.0I37347.

26. Reed S, Neuman H, Moscovich S, Glahn RP, Koren O, Tako E. Chronic Zinc Deficiency Alters Chick Gut Microbiota Composition and Function. Nutrients 2015;7(I2):9768-84. DOI: 10.3390/nu7I 25497.

\section{AUTHOR'S CONTRIBUTION}

Following authors have made substantial contributions to the manuscript as under:

MD: Conception and study design, acquisition of data, drafting the manuscript, approval of final version to be published

RN: Acquisition of data, drafting the manuscript, approval of final version to be published

NS: Analysis and interpretation of data, drafting the manuscript, approval of final version to be published

MA: Acquisition, analysis and interpretation of data, critical review, approval of final version to be published

JA: Analysis and interpretation of data, drafting the manuscript, approval of final version to be published

Authors agree to be accountable for all aspects of the work in ensuring that questions related to the accuracy or integrity of any part of the work are appropriately investigated and resolved.

CONFLICT OF INTEREST
Authors declared no conflict of interest
GRANT SUPPORT AND FINANCIAL DISCLOSURE
This research work is partially supported by grant to first author by
the Office of Research, Innovation and Commercialization (ORIC),
Khyber Medical University, Peshawar, Pakistan.

\section{CONFLICT OF INTEREST}

Conflict of interest

This research work is partially supported by grant to first author by Khyber Medical University, Peshawar, Pakistan.

\section{DATA SHARING STATEMENT}

The data that support the findings of this study are available on request from the corresponding author. The data are not publicly available due to privacy or ethical restrictions.

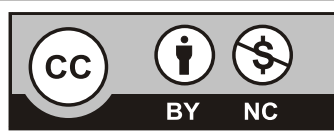

This is an Open Access article distributed under the terms of the Creative Commons Attribution-Non Commercial 2.0 Generic License.

KMUJ web address: www.kmuj.kmu.edu.pk

Email address: kmuj@kmu.edu.pk 